\title{
Timing of Bariatric Surgery in Liver Transplant Recipients; A Comprehensive Systematic Review
}

\section{Thamer Kassim, Haitam Buaisha, Jonathan Gapp, Jahnavi Koppala, Ryan Walters, Rajani Rangray* and Sandeep Mukherjee}

Creighton University Medical Center, USA

*Corresponding Author: Rajani Rangray, Creighton University Medical Center, USA.
Received: January 06, 2021

Published: Febuary 17, 2021

(C) All rights are reserved by Rajani Rangray., et al.

\begin{abstract}
Background and Objectives: Rising obesity rates with an associated increased prevalence of nonalcoholic steatohepatitis (NASH) have become a leading cause of end stage liver disease (ESLD). This excess weight poses particular challenges in the care of liver transplant (LT) patients. We conduct a systematic review to investigate the most safe and feasible timing to undergo bariatric surgery in patients with end-stage liver disease undergoing liver transplant.

Methods: We performed a literature search on studies reporting BS associated with LT in adults. There was no enough comparable information to conduct a metanalysis. A systematic review was conducted.

Results: BS prior to LT: Eight retrospective studies with small groups of patients (n: 6 - 78) examined BS prior to LT. Most of these studies suggest that this approach is relatively safe, efficacious and carries a low complication rate. However, one larger study suggested that BS (mainly RGYB) prior to LT may be associated with higher delisting rates or deaths prior to LT. Simultaneous BS and LT: Most of the reported studies are limited in their sample size and follow up duration. They showed improvement in obesity related complications and resulted in effective and durable weight loss. BS after LT: Six studies were reported with overall 43 patient. Improvement in the metabolic comorbidities following BS were noted and no early mortality was reported with respect to BS. Overall complication rates were higher than nontransplant population. Effects on immunosuppression were minimal with no reported graft rejection.
\end{abstract}

Conclusion: BS performed before, after, or simultaneously to LT seems to be an acceptable option for obese patients with ESLD.

Keywords: Systematic Review; Liver Transplantation; Bariatric Surgery

\section{Abbreviations}

BS: Bariatric Surgery; LT: Liver Transplant; LSG: Laparoscopic Sleeve Gastrectomy; SG: Sleeve Gastrectomy; RYGB: Roux-En-Y Gastric Bypass; GB: Gastric Banding

\section{Introduction}

The epidemic of obesity defined as BMI of more than $30 \mathrm{~kg} / \mathrm{m}^{2}$ is on the rise worldwide. The projected worldwide prevalence by
2030 for overweight individuals will be $38 \%$ and obese individuals will be $20 \%$. In the United States, the prevalence has been increasing over the last decade. It is estimated that by $2030,>50 \%$ of US population will have a BMI of $>30 \mathrm{~kg} / \mathrm{m}^{2}$ [1]. Paralleling obesity, prevalence of nonalcoholic fatty liver disease (NAFLD) and nonalcoholic steatohepatitis (NASH) is also on the rise. The prevalence of NAFLD is has increased from 15\% in 2005 to 25\% in 2010 and similarly, the prevalence rate of NASH has increased from $33 \%$ to 
$59.1 \%$ in same time frame. NASH will be the leading cause of liver transplantation (LT) in the next decade [2]. Liver transplant is challenging in these subgroups of patients due to several issues. The American Association for the Study of Liver Disease (AASLD) and the American Society of Transplantation considers obesity with pre- liver transplant $\mathrm{BMI}>40 \mathrm{~kg} / \mathrm{m}^{2}$ as a relative contraindication for liver transplantation [3]. There is increased risk of postliver transplant mortality for patients with a BMI $\geq 40$ at 30 days as well as at 1 and 2 years after transplantation in a recent systematic review and meta-analysis [4]. There is also increased prevalence of post- transplant obesity in 30\%-40\% of liver transplant recipients within the first 5 years after transplantation [5], associated with immunosuppressive medication, development of metabolic syndrome and recurrence of chronic liver disease. This growing burden of obesity, development of liver disease and the challenges associated before and after liver transplant warrants the urgent need for aggressive weight loss methods. Medical weight loss approach has been effective in decreasing $10 \%$ body weight; we review the different bariatric surgeries with respect to liver transplant.

The three main bariatric surgical methods, consisting of Rouxen-Y Gastric Bypass (RYGB), Gastric Banding (GB) and Sleeve Gastrectomy (SG), have not been routinely used for liver disease before or after liver transplant or for cirrhotic patients. Multiple studies have shown the safety of these surgeries in non-cirrhotic patients. A recent study has shown resolution of NASH in $85 \%$ of patients, confirmed by histological diagnosis at 1 year after bariatric surgery in addition to weight loss, improvement in liver chemistries such as ALT and NAFLD score [6]. The RYGB is the gold standard bariatric surgery but associated with higher rate of complications [7]. This is worrisome especially in the background of liver disease patients who have higher morbidity associated with any type of surgical interventions. There is also concern regarding the absorption of immunosuppressive medications in RYGB patients due to the altered anatomy [8] and close monitoring of the immunosuppressive medications is required. Contrarily, SG has not been associated with any difference in absorption of the immunosuppressive therapy and no dosage changes are needed [9]. The indications of bariatric surgery in general populations are clear: $\mathrm{BMI} \geq 40$, or more than 100 pounds overweight, $\mathrm{BMI} \geq 35$ and at least one or more obesityrelated co-morbidities such as type II diabetes (T2DM), hypertension, sleep apnea and other respiratory disorders, non-alcoholic fatty liver disease, osteoarthritis, lipid abnormalities, gastrointes- tinal disorders, or heart disease and inability to achieve healthy weight loss sustained for a period of time with prior weight loss efforts as per the American Society for Metabolic and Bariatric Surgery. The question of extrapolating these indications for liver transplant patients is not clear and very little data are available for advanced liver disease patients. Similarly, the timing of this bariatric surgery in peri liver transplant patient is debatable, whether to perform before simultaneously or after liver transplant. In this systematic review we explore the different types of bariatrics surgeries and the adequate timing of these surgeries with regards to liver transplant.

\section{Materials and Methods}

Literature search: A systemic literature review was performed according to the Preferred Reporting Items for Systematic Reviews and Meta-Analyses (PRISMA) statement. We searched PubMed, Scopus, Google scholar, CINAHL and Cochrane Library databases for studies reporting BS associated with LT in adults submitted in English language only. No limitation regarding the timing of the bariatric surgery in relation to the liver transplant surgery (before, after, or simultaneously). The search was conducted in January 2019 and was not limited to any date range. We used the following keywords in various combinations; "liver transplantation", "bariatric surgery”, "Gastric Bypass", "gastric banding", "Gastroplasty”, and "Gastrectomy." Bibliography of selected manuscripts and review articles was also manually searched for additional studies which were not identified in the original search.

Study selection: Two authors (TK and JG) independently screened titles and abstracts to identify studies for full text review. 35 potentially relevant article was analyzed for eligibility for systematic review and metanalysis. Studies with 3 or more patients were included for potential metanalysis (refer to the attached table) and information was extracted using a standardized data extraction.

\section{Results and Discussion}

BS prior to LT

Surgical treatment aims to improve accessibility and outcomes of LT. Morbid obesity remains a relative contraindication for LT per AASLD and obese patients suffer from limited access to LT $[10,11]$. BS in the setting of ESLD have been shown to be effective in weight loss prior to LT [10]. There are eight studies [12-19] to date which examined the role of BS prior to LT. These were retrospective stud- 


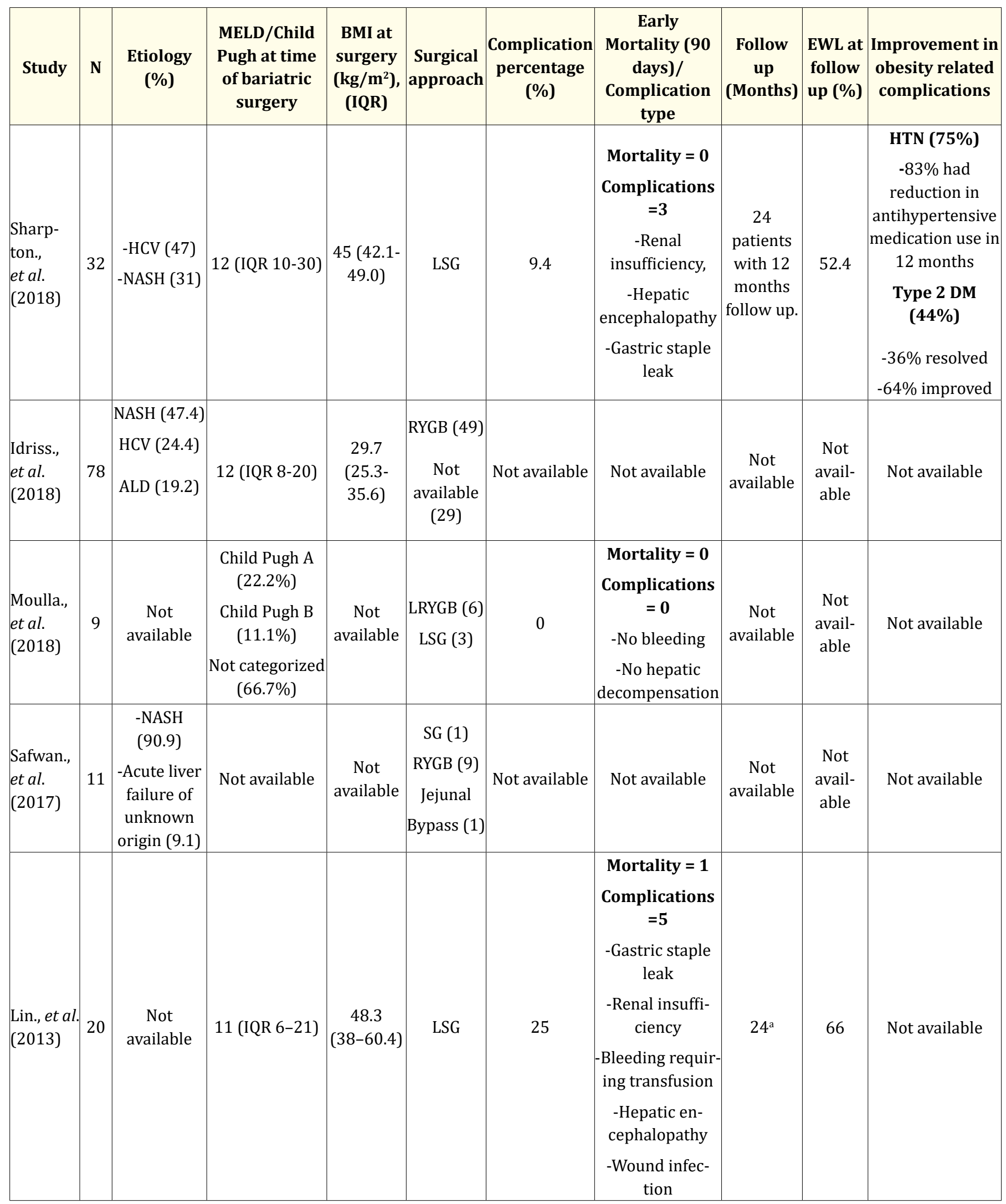




\begin{tabular}{|c|c|c|c|c|c|c|c|c|c|c|}
\hline $\begin{array}{l}\text { Rebibo., } \\
\text { et al. } \\
(2013)\end{array}$ & 13 & $\begin{array}{c}\text { NASH (93) } \\
\text { NASH \& ALD } \\
(7)\end{array}$ & $\begin{array}{l}\text { MELD } 7 \text { (7-8) } \\
\text { Child Pugh A } \\
\quad(100 \%)\end{array}$ & $\begin{array}{c}46.3 \\
(38.1- \\
56.8)\end{array}$ & LSG & 7.7 & $\begin{array}{c}\text { Mortality = 0 } \\
\text { Complications } \\
=\mathbf{1} \\
\text {-Intrabdomi- } \\
\text { nal hematoma } \\
\text { resolved with no } \\
\text { surgical inter- } \\
\text { vention (1) }\end{array}$ & \begin{tabular}{|c|}
-11 \\
patients \\
followed \\
up at 6 \\
months \\
-5 patients \\
followed \\
up at 12
\end{tabular} & $\begin{array}{l}61.9(6 \\
\text { months }) \\
73.4(12 \\
\text { months })\end{array}$ & Not available \\
\hline $\begin{array}{l}\text { Shimzu., } \\
\text { et al. } \\
(2012)\end{array}$ & 23 & $\begin{array}{c}\text { Not } \\
\text { available }\end{array}$ & $\begin{array}{l}\text { Child Pugh A } \\
\qquad(95.7 \%) \\
\text { Child Pugh B } \\
\quad(4.3 \%)\end{array}$ & $\begin{array}{c}47.1 \\
(37.8- \\
56.3)\end{array}$ & $\begin{array}{c}\text { LSG (8) } \\
\text { GB (1) } \\
\text { RYGB (14) }\end{array}$ & 34.8 & $\begin{array}{c}\text { Mortality = 1 } \\
\text { (LSG) } \\
\text { Complications } \\
=\mathbf{8} \\
\text { (28.6\% LGYB), } \\
\text { (37.5\% LSG) } \\
\text {-Gastrojejunal } \\
\text { anastomotic leak } \\
\text {-Gastrojejunal } \\
\text { stricture } \\
\text {-Infected } \\
\text { hematoma } \\
\text {-Gastric staple } \\
\text { line leak } \\
\text {-Gastric stricture } \\
\text {-GI bleeding } \\
\text {-Pneumonia }\end{array}$ & $\begin{array}{c}-18 \\
\text { patients } \\
\text { with } 12 \\
\text { months } \\
\text { follow up. } \\
-15 \\
\text { patients } \\
\text { with mean } \\
37 \text { months } \\
\text { follow up }\end{array}$ & 67.5 & \begin{tabular}{|c|} 
HTN \\
-68.7\% remission \\
or improved) \\
Type 2 DM \\
$-86.7 \%$ \\
remission or \\
improved) \\
DLP \\
-66.7\% remission \\
or improved
\end{tabular} \\
\hline
\end{tabular}

Table 1: Summary of bariatric surgeries performed in patient with cirrhosis prior to receiving liver transplantation.

Abbreviations: MELD: Model of End-Stage Liver Disease; BMI: Body Mass Index; IQR: Interquartile Range; NASH: Nonalcoholic Steatohepatitis; HCV: Hepatitis C Virus; HBV: Hepatitis B Virus; ALD: Alcoholic liver disease; RYGB: Roux-en-Y Gastric Bypass; LSG: Laparoscopic Sleeve Gastrectomy; SG: Sleeve Gastrectomy.

$$
\text { a: Mean. }
$$




\begin{tabular}{|c|c|c|c|c|c|c|c|c|c|c|}
\hline Study & $\mathbf{N}$ & Etiology (\%) & $\begin{array}{l}\text { MELD/ } \\
\text { Child } \\
\text { Pugh }\end{array}$ & $\begin{array}{c}\text { BMI at } \\
\text { surgery } \\
\left(\mathrm{kg} / \mathrm{m}^{2}\right), \\
(\mathrm{IQR})\end{array}$ & $\begin{array}{c}\text { Surgical } \\
\text { approach }\end{array}$ & $\begin{array}{c}\text { Complication } \\
\text { percentage } \\
(\%)\end{array}$ & $\begin{array}{l}\text { Early Mortality } \\
(90 \text { days }) / \text { Com- } \\
\text { plication type }\end{array}$ & 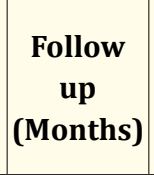 & $\begin{array}{c}\text { EWL at } \\
\text { follow up, } \\
\%\end{array}$ & $\begin{array}{c}\text { Improvement } \\
\text { in obesity } \\
\text { related } \\
\text { complications }\end{array}$ \\
\hline $\begin{array}{l}\text { Zamora- } \\
\text { Valdes., et } \\
\text { al. (2018) }\end{array}$ & 29 & $\begin{array}{c}\text { NAFLD alone } \\
\text { (52.7) } \\
\text { NAFLD + AAD } \\
(20.7) \\
\text { NAFLD + AIH } \\
(6.9) \\
\text { AAD (3.45) } \\
\text { HHT (3.45) } \\
\text { HCV (3.45) } \\
\text { Allograft failure } \\
(3.45) \\
\text { FHF (3.45) } \\
\text { PBC (3.45) }\end{array}$ & $32^{\mathrm{a}}$ & $47^{\mathrm{a}}$ & SG & 44.8 & $\begin{array}{c}\text { Mortality = 2 } \\
\text { Complications } \\
=\mathbf{1 0} \\
\text {-Bleeding requiring } \\
\text { reoperation (3) } \\
\text {-On HD with } \\
\text { persistence of renal } \\
\text { failure after LT (3) } \\
\text {-HAT (2) } \\
\text {-Gastric staple line } \\
\text { leak (1) } \\
\text {-Mucor mycosis } \\
\text {-excised (1) }\end{array}$ & 36 & $\begin{array}{c}36.3-12 \\
\text { months } \\
(\mathrm{p}=0.049) \\
34.4-24 \\
\text { months } \\
(\mathrm{p}=0.562) \\
\\
36.9-36 \\
\text { months } \\
(\mathrm{p}=0.81)\end{array}$ & $\begin{array}{c}\text { Type } 2 \text { DM } \\
\text { HTN } \\
\text { Hepatic } \\
\text { Steatosis }\end{array}$ \\
\hline $\begin{array}{l}\text { Nesher., } \\
\text { et al. } \\
(2017)\end{array}$ & 3 & $\begin{array}{c}\text { NASH (33.3) } \\
\text { NASH + HCV } \\
(33.3) \\
\text { NASH + Wilson } \\
(33.3)\end{array}$ & $\begin{array}{c}24(23- \\
24)\end{array}$ & & SG & $33.3 \%$ & $\begin{array}{c}\text { Mortality = 0 } \\
\text { Complications = 1 } \\
\text {-Biliary } \\
\text { anastomotic leak \& } \\
\text { transient AKI }\end{array}$ & $\begin{array}{c}13^{\mathrm{b}} \text { (range } \\
3-24)\end{array}$ & $\begin{array}{c}27.9-13 \\
\text { months }\end{array}$ & $\begin{array}{c}\text { HTN } \\
\text {-Resolved in } \\
100 \% \\
\text { Type 2 DM } \\
\text {-Resolved in } \\
\quad 100 \% \\
\end{array}$ \\
\hline $\begin{array}{l}\text { Heim- } \\
\text { bach., et } \\
\text { al. (2013) }\end{array}$ & 7 & $\begin{array}{c}\text { NASH (57.1) } \\
\text { NASH+HCV } \\
(14.3) \\
\text { NASH+AAD } \\
\quad(14.3) \\
\text { HHT (14.3) }\end{array}$ & $\begin{array}{c}32(11- \\
40)\end{array}$ & $\begin{array}{c}48 \\
(39-52)\end{array}$ & SG & 42.9 & $\begin{array}{c}\text { Mortality =0 } \\
\text { Complications =3 } \\
\text {-Gastric staple line } \\
\text { leak (1) } \\
\text {-HAT (1) } \\
\text {-Steroid resistant } \\
\text { rejection (1) }\end{array}$ & $\begin{array}{c}17 \text { (range } \\
8-33 \text { ) }\end{array}$ & $\begin{array}{c}\text { Not } \\
\text { available }\end{array}$ & $\begin{array}{c}\text { Type } 2 \text { DM } \\
\text {-Improved or } \\
\text { resolved in } \\
100 \% \\
\text { Hepatis } \\
\text { steatosis } \\
\text {-0/7 had } \\
\text { findings of } \\
\text { steatosis }\end{array}$ \\
\hline
\end{tabular}

Table 2: Summary of studies on bariatric surgery performed simultaneously with liver transplantation.

Abbreviations: MELD: Model of End-Stage Liver Disease; BMI: Body Mass Index; IQR: Interquartile Range; NAFLD: Non-Alcoholic Fatty Liver Disease; NASH: Non-Alcoholic Steatohepatitis; FHF- Fulminant Hepatic Failure; PBC: Primary Biliary Cirrhosis; AAD: Alpha 1 Antitrypsin Deficiency; HAT: Hepatic Artery Thrombosis; HHT: Hereditary Hemorrhagic Telangiectasia; HCV - Hepatitis C Virus; AIH: Autoimmune Hepatitis; SG: Sleeve Gastrectomy; AKI: Acute Kidney Injury.

$$
\begin{gathered}
\text { a: Mean. } \\
\text { b: Median. }
\end{gathered}
$$




\begin{tabular}{|c|c|c|c|c|c|c|c|c|c|c|}
\hline Study & $\mathbf{N}$ & $\begin{array}{c}\text { Etiology } \\
(\%)\end{array}$ & $\begin{array}{l}\text { MELD/ } \\
\text { Child } \\
\text { Pugh }\end{array}$ & $\begin{array}{c}\text { BMI at } \\
\text { surgery } \\
\left(\mathrm{kg} / \mathrm{m}^{2}\right), \\
(\mathrm{IQR})\end{array}$ & $\begin{array}{c}\text { Surgical } \\
\text { approach }\end{array}$ & $\begin{array}{c}\text { Complication } \\
\text { percentage } \\
(\%)\end{array}$ & \begin{tabular}{|c} 
Early \\
Mortality (90 \\
days)/ \\
Complication \\
type
\end{tabular} & $\begin{array}{l}\text { Follow up } \\
\text { (Months) }\end{array}$ & $\begin{array}{c}\text { EWL at } \\
\text { follow up, } \\
\%\end{array}$ & $\begin{array}{l}\text { Improvement in } \\
\text { obesity related } \\
\text { complications }\end{array}$ \\
\hline $\begin{array}{l}\text { Osseis., et } \\
\text { al. (2018) }\end{array}$ & 6 & $\begin{array}{c}\text { HCV (1) } \\
\text { ALD (2) } \\
\text { NASH (1) } \\
\text { HCV + ALD } \\
(1) \\
\text { ALD + NASH } \\
(1)\end{array}$ & $\begin{array}{c}\text { Not } \\
\text { available }\end{array}$ & $\begin{array}{c}43.7 \\
(38-44.9)\end{array}$ & $\begin{array}{l}\text { LSG (3) } \\
\text { SG (3) }\end{array}$ & 17 & $\begin{array}{c}\text { Mortality }=1 \\
\text {-Death after } 19 \\
\text { months } \\
\text { Complications= } \\
1 \\
\text {-Gastric staple } \\
\text { line leak, } \\
\text { multi-organ } \\
\text { failure, death }\end{array}$ & $\begin{array}{c}37.2^{\mathrm{b}}: \\
\text { Range } \\
(13-101 \\
\text { months })\end{array}$ & $\begin{array}{c}76^{\text {b }} \text { : Range } \\
(25-119)\end{array}$ & $\begin{array}{c}\text { DM } \\
-100 \% \text { improved or } \\
\text { resolved } \\
\text {-HTN } \\
50 \% \text { didn't require } \\
\text { medications 1 year } \\
\text { after SG } \\
\text {-OSA } \\
66.7 \% \text { improved }\end{array}$ \\
\hline $\begin{array}{l}\text { Tsam- } \\
\text { alaidze., } \\
\text { et al. } \\
(2018)\end{array}$ & 12 & $\begin{array}{c}\text { HCV (5) } \\
\text { NASH (4) } \\
\text { AIH (1) } \\
\text { ALD (1) } \\
\text { Cryptogenic } \\
\text { (1) }\end{array}$ & $\begin{array}{c}\text { Not } \\
\text { available }\end{array}$ & $45.3^{\mathrm{a}}$ & LSG & 33.3 & $\begin{array}{c}\text { Mortality = 0 } \\
\text { Complications } \\
\mathbf{=} \mathbf{4} \\
\text {-Sleeve dilation } \\
\text { for } \\
\text { malnutrition } \\
(2) \\
\text {-Gastrostomy } \\
\text { tube placement } \\
\text { for } \\
\text { malnutrition } \\
(1) \\
\text {-Late drain } \\
\text { removal (1) }\end{array}$ & $25.3^{\mathrm{a}}$ & $\begin{array}{l}50 \text { (at } 12 \\
\text { months) }\end{array}$ & $\begin{array}{c}\text { Type } 2 \text { DM } \\
-44 \% \text { resolution } \\
\text { DLP } \\
43 \% \text { resolution } \\
\text { HTN } \\
-27 \% \text { resolution } \\
\text { OSA } \\
-43 \% \text { resolution }\end{array}$ \\
\hline $\begin{array}{l}\text { Yemini., et } \\
\text { al. (2018) }\end{array}$ & 4 & $\begin{array}{c}\text { Not } \\
\text { available }\end{array}$ & $\begin{array}{c}\text { Not } \\
\text { available }\end{array}$ & $\begin{array}{c}41 \\
(45-48)^{*}\end{array}$ & $\begin{array}{c}\text { LSG } \\
\text { LRYGB }\end{array}$ & 25 & $\begin{array}{c}\text { Mortality }=\mathbf{0} \\
\text { Complications } \\
=\mathbf{1} \\
\text {-Anastomotic } \\
\text { leak requiring } \\
\text { reoperation }\end{array}$ & $12^{*}$ & $78 \%{ }^{*}$ & Not available \\
\hline $\begin{array}{l}\text { Kho- } \\
\text { raki., et al. } \\
(2016)\end{array}$ & 5 & $\begin{array}{c}\text { NASH (3) } \\
\text { PBC (1) } \\
\operatorname{HCV}(1)\end{array}$ & $\begin{array}{c}\text { Not avail- } \\
\text { able }\end{array}$ & $47.2^{\mathrm{a}}$ & LSG & 40 & $\begin{array}{c}\text { Mortality }=\mathbf{0} \\
\text { Complications } \\
=\mathbf{1}\end{array}$ & $33.2^{\mathrm{a}}$ & $\begin{array}{l}43 \text { (at } 24 \\
\text { months) }\end{array}$ & $\begin{array}{c}\text { Type } 2 \text { DM } \\
-60 \% \text { achieved } \\
\text { resolution } \\
\text { HTN } \\
-40 \% \text { achieved } \\
\text { resolution and didn't } \\
\text { require medications. }\end{array}$ \\
\hline
\end{tabular}




\begin{tabular}{|c|c|c|c|c|c|c|c|c|c|c|}
\hline $\begin{array}{l}\text { Lin., et al. } \\
(2013)\end{array}$ & 9 & $\begin{array}{c}\text { Not } \\
\text { available }\end{array}$ & $\begin{array}{c}\text { Not } \\
\text { available }\end{array}$ & $\begin{array}{c}41(38- \\
44)\end{array}$ & $\begin{array}{l}\text { LSG (8) } \\
\text { SG (1) }\end{array}$ & 33.3 & $\begin{array}{c}\text { Mortality = 0 } \\
\text { Complications } \\
=\mathbf{3} \\
\text {-Incisional } \\
\text { hernia (1) } \\
\text {-Biliary leak (1) } \\
\text {-Dysphagia } \\
\text { requiring } \\
\text { conversion to } \\
\text { RYGB (1) }\end{array}$ & 3-36 & $\begin{array}{l}55 \text { (at } 6 \\
\text { months) }\end{array}$ & $\begin{array}{c}\text { DM } \\
\text {-Improved } \\
\text { DLP } \\
\text {-Improved }\end{array}$ \\
\hline $\begin{array}{l}\text { Al-No- } \\
\text { waylati., } \\
\text { et al. } \\
(2013)\end{array}$ & 7 & $\begin{array}{c}\text { HCV (4) } \\
\text { ALD (1) } \\
\text { Jejunoileal } \\
\text { Bypass (1) } \\
\text { Hemangio- } \\
\text { endo } \\
\text {-thelioma } \\
\text { (1) }\end{array}$ & $\begin{array}{c}\text { Not } \\
\text { available }\end{array}$ & $44.3^{\mathrm{a}}$ & RYGB & 42.9 & $\begin{array}{c}\text { Mortality = 2 } \\
\text { Complications } \\
=\mathbf{3} \\
\text {-Incisional } \\
\text { hernia (2) } \\
\\
\text {-Malnutrition } \\
\text { requiring re- } \\
\text { versal of RYGB } \\
\text { (1) }\end{array}$ & $59.1^{\mathrm{a}}$ & $\begin{array}{c}\text { BMI } 44.3 \\
\rightarrow 26 \mathrm{~kg} / \\
\mathrm{m}^{2}(59 \\
\text { months })\end{array}$ & $\begin{array}{c}\text { Type } 2 \text { DM } \\
\text {-Improved } \\
\text { DLP } \\
\text {-Improved }\end{array}$ \\
\hline
\end{tabular}

Table 3: Summary of studies on bariatric surgery performed after liver transplantation.

Abbreviations: MELD: Model of End-Stage Liver Disease; BMI: Body Mass Index; NASH: Non-Alcoholic Steatohepatitis; ALD: Alcoholic liver disease; RYGB: Roux-en-Y Gastric Bypass; LSG: Laparoscopic Sleeve Gastrectomy; SG - Sleeve Gastrectomy; GB: Gastric Band HCV - Hepatitis C Virus; PBC: Primary Biliary Cirrhosis; AIH: Autoimmune Hepatitis; ALD: Alcoholic liver disease; OSA: Obstructive Sleep Apnea; DLP: Dyslipidemia; EWL: Excess Weight Loss; LT: Liver Transplant; BS: Bariatric Surgery.

$$
\begin{aligned}
& \text { a: Mean. } \\
& \text { b: Median. }
\end{aligned}
$$

*: Data is represented for all cohort (LT patients were a subgroup of a bigger cohort).

ies with small groups of patients (n of 6-78) [12-19] which examined different aspects of BS prior to LT. There were two case reports, Chmura., et al. [20] and Taneja., et al. [21], reporting RNYGB and LSG, respectively, prior to receiving LT (Supplemental table).

In one study, Sharpton., et al. [12] evaluated 32 patients which were known to have cirrhosis predominantly from hepatitis $\mathrm{C}$ (47\%) and NASH (31\%) for LT. These patients underwent LSG prior to the procedure to achieve weight loss. In this cohort, the average BMI prior to SG was $45 \mathrm{~kg} / \mathrm{m}^{2}$ (IQR $42.1-49.0 \mathrm{~kg} / \mathrm{m}^{2}$ ). The median time between LSG and LT was 22 months (IQR, 14 - 88 months). Fourteen patients underwent LSG then LT and MELD laboratory score at the time of LT was 15 (IQR 12 - 28). The median EWL at last follow up (12 months) after LSG was 52.4\%. Nine patients were delisted due to low MELD score and improved liver markers. Three patients either did not follow up for transplant evaluation or transferred care to another transplant center. One patient had a surgical related complication of gastric leak and was precluded from LT. There were two patients who were delisted due to psychological reasons and two patients died from advanced liver disease 


\begin{tabular}{|c|c|c|c|c|c|c|c|c|c|c|}
\hline & Age & Gender & $\begin{array}{c}\text { Etiology } \\
(\%)\end{array}$ & $\underset{\text { Pugh }}{\text { MELD/Child }}$ & $\begin{array}{c}\text { BMI at } \\
\text { surgery (kg/ } \\
\text { m²), (IQR) }^{2}\end{array}$ & $\begin{array}{c}\text { Surgical } \\
\text { approach }\end{array}$ & $\begin{array}{c}\text { Surgery } \\
\text { associated } \\
\text { complication }\end{array}$ & $\begin{array}{l}\text { Follow up } \\
\text { (Months) }\end{array}$ & $\begin{array}{c}\text { Weight } \\
\text { reduction at } \\
\text { follow up (\%) }\end{array}$ & $\begin{array}{l}\text { Improvement } \\
\text { in obesity } \\
\text { related } \\
\text { complications }\end{array}$ \\
\hline \multicolumn{11}{|l|}{$\begin{array}{l}\text { BS prior } \\
\text { to LT } \\
\end{array}$} \\
\hline $\begin{array}{l}\text { Chmura., } \\
\text { et al. } \\
(2015)\end{array}$ & 56 & Female & $\begin{array}{c}\text { NASH + } \\
\text { ALD }\end{array}$ & Not available & 50.9 & RYGB & \begin{tabular}{|c|}
-Intraperitone- \\
al hemorrhage \\
requiring \\
revision \\
surgery \\
-AKI \\
requiring \\
temporary \\
dialysis \\
\end{tabular} & 36 & 50.1 & $\begin{array}{l}\text { Type } 2 \text { DM } \\
\text {-Complete } \\
\text { remission }\end{array}$ \\
\hline $\begin{array}{l}\text { Taneja., et } \\
\text { al. (2013) }\end{array}$ & 29 & Male & $\mathrm{NASH}$ & $\begin{array}{l}\text { 14/ Child } \\
\text { Pugh B }\end{array}$ & 55.3 & LSG & None & 6 & 36.3 & Not available \\
\hline \multicolumn{11}{|l|}{$\begin{array}{l}\text { Simulta- } \\
\text { neous BS } \\
\& \text { LT } \\
\end{array}$} \\
\hline $\begin{array}{l}\text { Kumar., et } \\
\text { al. (2017) }\end{array}$ & 58 & Female & $\mathrm{NASH}$ & $\begin{array}{l}\text { 27/Child } \\
\text { Pugh C } \\
\end{array}$ & 38.8 & SG & Not available & 2 & 25 & Not available \\
\hline $\begin{array}{l}\text { Guerrero } \\
\text { Pérez., } \\
\text { et al. } \\
(2017)\end{array}$ & 54 & Male & $\mathrm{HCV}$ & $\begin{array}{l}\text { 30/Child } \\
\text { Pugh C }\end{array}$ & 37.7 & SG & $\begin{array}{c}\text {-ARDS } \\
\text {-CMV infection } \\
\text {-C-Diff infection }\end{array}$ & 24 & 38 & $\begin{array}{c}\text { HTN } \\
\text {-Decrease in } \\
\text { total } \\
\text { medications } \\
\text { DLP } \\
\text {-Stable } \\
\text { OSA } \\
\text {-Resolved }\end{array}$ \\
\hline $\begin{array}{l}\text { Tariciotti., } \\
\text { et al. } \\
(2016)\end{array}$ & 53 & Female & $\mathrm{HCV}$ & 14 & 38 & SG & None & 5 & Not available & $\begin{array}{l}\text { Type } 2 \text { DM } \\
\text {-Complete } \\
\text { remission }\end{array}$ \\
\hline $\begin{array}{l}\text { Campsen., } \\
\text { et al. } \\
(2008)\end{array}$ & 28 & Female & $\mathrm{AIH}$ & Not available & 42 & GB & None & 6 & 45 & $\begin{array}{c}\text { HTN } \\
\text {-No longer } \\
\text { required } \\
\text { medications } \\
\text { OSA } \\
\text {-Symptoms } \\
\text { improved } \\
\text { Venous stasis } \\
\text {-Improved }\end{array}$ \\
\hline
\end{tabular}




\begin{tabular}{|c|c|c|c|c|c|c|c|c|c|c|}
\hline \begin{tabular}{|l} 
BS after \\
LT \\
\end{tabular} & & & & & & & & & & \\
\hline $\begin{array}{l}\text { Lainas., et } \\
\text { al. (2018) }\end{array}$ & 39 & Female & FHF & Not available & 43.7 & SPSG & None & 24 & EWL - 79.5 & \begin{tabular}{|c} 
HTN \\
-Improved, \\
requires one \\
medication \\
instead of two \\
OSA \\
-Resolved, \\
doesn't need \\
CPAP
\end{tabular} \\
\hline $\begin{array}{l}\text { Pajecki., et } \\
\text { al. (2014) }\end{array}$ & 33 & Female & $\mathrm{AIH}+\mathrm{HVC}$ & 31 & $\begin{array}{c}37.1 \text { (at time } \\
\text { of LT) }\end{array}$ & LSG & None & 5 & EWL - 75 & $\begin{array}{c}\text { HTN } \\
\text {-Improved, } \\
\text { doesn't need } \\
\text { medications } \\
\text { Type 2 DM } \\
\text {-Improved, } \\
\text { doesn't need } \\
\text { medications }\end{array}$ \\
\hline $\begin{array}{l}\text { Elli., et al. } \\
(2012)\end{array}$ & 62 & Female & $\mathrm{HCV}$ & Not available & 53 & SG & None & 3 & $\begin{array}{c}\text { BMI } 53 \rightarrow 48 \\
\mathrm{~kg} / \mathrm{m}^{2}\end{array}$ & Not available \\
\hline $\begin{array}{l}\text { Gentiles- } \\
\text { chi., et al. } \\
(2009)\end{array}$ & 57 & Male & $\mathrm{HCV}$ & Not available & 54 & BPD & None & 6 & 21.4 & $\begin{array}{c}\text { Type } 2 \text { DM } \\
\text { HTN } \\
\text { OSA }\end{array}$ \\
\hline \begin{tabular}{|l|} 
Butte., et \\
al. (2007)
\end{tabular} & 63 & Male & NASH & Not available & 41 & SG & None & 6 & $\begin{array}{c}\text { BMI } 41 \rightarrow 38 \\
\mathrm{~kg} / \mathrm{m}^{2}\end{array}$ & Type 2 DM \\
\hline $\begin{array}{l}\text { Tichan- } \\
\text { sky., et al. } \\
\text { (2005) }\end{array}$ & 49 & Female & HCV & & 54 & RYGB & None & 4 & $\begin{array}{c}\text { BMI } 54 \rightarrow 43 \\
\mathrm{~kg} / \mathrm{m}^{2}\end{array}$ & $\begin{array}{l}\text { Type } 2 \text { DM } \\
\text {-Complete } \\
\text { resolution } \\
\text { HTN } \\
\text {-Complete } \\
\text { resolution }\end{array}$ \\
\hline
\end{tabular}

Supplementary table: Case reports on timing of bariatric surgery and liver transplantation.

Abbreviations: MELD: Model of End-Stage Liver Disease; BMI: Body Mass Index; NASH: Non-Alcoholic Steatohepatitis; ALD: Alcoholic liver disease; FHF- Fulminant Hepatic Failure; RYGB: Roux-en-Y Gastric Bypass; LSG: Laparoscopic Sleeve Gastrectomy; SG - Sleeve Gastrectomy; SPSG: Single Port Sleeve Gastrectomy; GB: Gastric Band HCV - Hepatitis C Virus; BPD: Biliopancreatic Diversion; AIH: Autoimmune Hepatitis; ALD: Alcoholic liver disease; OSA: Obstructive Sleep Apnea; DLP: Dyslipidemia; EWL: Excess Weight Loss; LT:

Liver Transplant; BS: Bariatric Surgery.

complications. The authors had one patient still listed for LT prior to publishing their study. Over $50 \%$ of the cohort was Child Pugh B with median MELD score of 12 (IQR 10 - 13). All had decompen- sated liver disease and there was no liver related morbidity or mortality with SG. Median length of stay was three days and there were no reoperations or conversions from laparoscopic to open surgery. Sharpton., et al. showed that LSG prior to LT achieved excellent re- 
sults in weight loss in preparation for LT without surgically related morbidity and mortality, despite their cohort's advanced decompensated liver disease. They suggest that LSG prior to LT might improve outcomes in wait list time and graft survival, but further studies are needed to examine the outcomes on LT in patient receiving prior LSG and compare with non-surgical techniques for weight loss in transplant recipients [12].

In a similar but smaller retrospective study by Lin., et al. [13] 20 patients with cirrhosis with median MELD score of 11 (range 6 - 21) underwent LSG with an excess weight loss of $66 \%$ at 24 months. Twenty five percent of cirrhotic patients who underwent LT had complications in the form of organ insufficiency, bleeding requiring blood transfusion, or superficial wound infection. One patient had a staple line leak and subsequently developed a chronic fistula. The patient died four years later due to his underlying liver disease and surgery related complications. There was no 30 days mortality related to LSG. Seven patients underwent LT (1 patient combined LT and kidney transplant) after 16.6 months (SD +/- 14 months). The mean pre-transplant BMI was 31.4 with $31.9 \%$ pretransplant weight reduction. The authors concluded that LSG in patients awaiting transplant is feasible, well tolerated, and provides adequate excess weight loss. Lin., et al. did not examine outcomes in patients receiving LT or comparing them to those who received LT without having prior BS [13].

In a study by Shimzu., et al. [14] 23 patients (91.7\% Child-Pugh class A) having BS prior to LT were examined. Bariatric procedures included LRYGB $(n=14)$, LSG $(n=8)$ and GB $(n=1)$. There were 12 patients with known cirrhosis prior to the procedure and 11 with unknown cirrhosis. Significant weight loss was achieved with EWL of $67.4+/-30.9 \%$ reported in the $81.8 \%$ of patients following up at 12 months. They had a complication rate of $34.5 \%$ which was equally divided between LRYGB and LSG. These complications included, anastomotic leak, strictures, hemorrhage requiring blood transfusion, and infected hematoma. Although there was no 30-day mortality, one death of unknown cause was reported in the LSG group 9 months following the procedure. In their cohort, $86.7 \%$ of the patients with type 2 diabetes mellitus had improved glucose control and $66.7 \%$ achieved clinical remission. Around $70 \%$ percent of patients with hypertension and hyperlipidemia had clinical improvement. More importantly, 3 patients showed improved fibrosis where $2(8.7 \%)$ dropped 1 grade in fibrosis staging after a liver biopsy which was repeated in 24 months. Shimzu., et al. [14] concluded that the benefits of BS in patients with cirrhosis outweigh the risks as it can improve overall morbidities.

Moula., et al. [15] reported that there were no complications, liver decompensation, or 30-day mortality in nine patients undergoing BS (6 LRYGB and 3 LSG). Three patients were known to have cirrhosis prior to BS. Two patients were classified as two Child-Pugh A and one as Child-Pugh B. The authors concluded that BS is safe in patients with cirrhosis. Other smaller studies such as Rebibo., et al. [16] in thirteen cirrhotic patients (Child Pugh A) undergoing sleeve gastrectomy, and Takata., et al. [17] in six cirrhotic patients undergoing LSG (4 Child-Pugh A and 2 Child-Pugh B), demonstrate low complication rates, significant weight loss, and no cirrhosis associated morbidity or mortality. These two studies also support that BS in patients with cirrhosis may improve candidacy for transplantation.

The previous studies suggest that BS in patients with cirrhosis awaiting transplant is relatively safe with a low complication rate as well as effective for weight loss. However, outcomes after receiving liver transplant were not examined. Safwan., et al. [18] and more recently, Idriss., et al. [19] looked at the outcomes in liver recipients after receiving BS. Safwan., et al. examined 11 patients (MELD 28.4 +/- 6.7) receiving BS (9 RYGB, 1 SG, 1 JIB) and found similar graft survival and post-transplant complication rates among patients with prior BS in comparison to the general transplant population.

In a larger study by Idriss., et al. [19] 78 patients with cirrhosis receiving BS prior to LT were examined. The major BS was RYGB $(63 \%)$ and the median MELD score was 12 (8 - 20). Interestingly, transplant candidacy denial was lower for the BS group $(42.3 \%$ versus 55.8\%; $\mathrm{P}=0.05$ ) however the rate of LT de-listing or death was higher in the BS group compared to the concurrent cohort (33.3\% versus $10.1 \% ; \mathrm{P}=0.002$ ). Furthermore, the rate of LT from the time of listing was lower in the BS group (48.9\% versus $65.2 \%$; $\mathrm{P}=0.03$ ). The authors suggest that sarcopenia and malnourishment are associated with delisting or death, as $64.1 \%$ in the BS group versus $39 \%$ of patients in the concurrent cohort $(\mathrm{P}=<0.01)$ were malnourished by the validated Subjective Global Assessment tool (SGA) at the time of transplant evaluation. Yet, comorbidities and markers of decompensation were lower in the BS group compared to the concurrent cohort, as LT evaluation occurred at a median time of 7 years after BS. Interestingly, the rates of delisting were higher in patients receiving RYGB versus non-RYGB BS (44\% 
versus 16.7\%; $\mathrm{P}=0.04$ ) and the rate of transplantation was lower (23.4\% versus 61.1\%; $\mathrm{P}<0.01$ ). Patients having moderate to severe malnutrition by SGA were higher in the RGYB group compared to non RGYB BS (67\% versus 57\%; $\mathrm{P}=0.05)$. Survival after one year of LT was similar in both groups 85\% (95\% CI, 71\%-100\%) and so was survival after 3 years of LT. However, the median time to transplant was lower in the BS group (54 months (IQR, 25 - 122 months) compared to the concurrent cohort 60 months (IQR, 25 82 months). Also, MELD at LT was higher in the BS group 22.0 (14.5 - 33.0) versus 18.0 (13.0 - 24.0).

Data by Idriss., et al. [19] suggest that BS prior to LT may be associated with higher delisting rates or deaths prior to LT. One of the major strengths of this study, in addition to having a larger patient group, is that patients were followed for 7 years, between BS to LT evaluation, and followed patients up to 3 years after LT examining survival. Results by Idriss., et al. [19] and Safwan., et al. [18] challenge previous studies (reference) which concluded that BS prior to LT is a safe option and may be associated with improved candidacy for LT. Therefore, BS prior to transplantation is still an area of developing research and further studies are needed to assess its safety, feasibility, and survival outcomes prior to and after LT.

\section{Simultaneous BS and LT}

Simultaneous BS and liver transplant surgery was first reported by Campsen in 2008. Campsen., et al. [22] described a 42-yearold female who underwent gastric banding with a repeat LT due to chronic rejection. The patient's pre transplant BMI was 42 and suffered from diabetes, hypertension, obstructive sleep apnea. The authors report that the patient recovered well from the surgery and was discharged after eight days. At 6 months follow up, the patient had an EWL of $45 \%$. The patient no longer needed treatment for type 2 diabetes or hypertension and had resolution of OSA symptoms. Between 2014 and 2017, three case reports from India, Italy, and Spain [23-25] described simultaneous LT and sleeve gastrectomy to be a relatively safe procedure with no life-threatening complications and showed improved outcomes in obesity related comorbidities.

In a low evidence - small case series $(\mathrm{n}=3)$ by Nesher., et al. [26] three patients with a median pre-transplant BMI of 46.6 and MELD of 24 underwent simultaneous SG and LT. The etiology of cirrhosis was predominantly related to NASH, with mean estimated weight loss of $27.9 \%$ at a median follow up of 13 months. They also describe improved overall obesity related comorbidities and no hepatic steatosis on abdominal ultrasound in two out of three patients. These studies report no significant perioperative complications or surgically related mortality along with improved obesity related outcomes. However, they are limited by their sample size (total $\mathrm{n}=7$ ) and short term follow up (5 to 24 months).

In study at the Mayo clinic in 2013, Heimbach., et al. [27] compared outcomes in patients $(n=7)$ undergoing simultaneous SG and LT with a cohort who achieved weight loss via a noninvasive method ( $\mathrm{n}=37$ ). In the SG and LT group, NASH was a primary etiology for cirrhosis in 57\%. Two out of the three patients had NASH as a secondary etiology for cirrhosis $66.7 \%$. Age at transplant was similar in the noninvasive and SG groups, 50 (range 31 - 67) vs 48 (range 44 -60), respectively. MELD scores and BMI at transplantation were both higher in the SG group compared to the noninvasive group [MELD 32 (range 11 - 40) vs 19(range 8 - 35) and BMI 48 (range 39 - 52) vs 33 (range 28 - 40)]. Mean BMI at last follow up in the SG group (17, range 18 - 33 months) vs noninvasive group (35, range 8 - 61 months) was 29 (23 - 35) and 36 (25 - 45) respectively. Obesity related comorbidities including HTN, DM and hepatic steatosis, were significantly improved in the SG group compared to the noninvasive cohort. The SG cohort had no intraoperative complications or surgery related mortality, but three patients had postoperative complications (gastric staple line leak, excess weight loss, and steroid resistant rejection). The authors concluded that $\mathrm{SG}$ at the time of LT is a procedure that carries risk but is feasible in carefully selected individuals [27].

Zamora-Valdes., et al. [28] updated their study at Mayo clinic in 2018 with an increase in sample size. Forty-five patients underwent LT alone vs 29 undergoing SG and LT. NAFLD was present in $48.9 \%$ of the overall cohort with higher prevalence in the LT+ SG cohort compared to LT alone $76.9 \%$ vs $44.4 \%$ respectively. Overall survival was better in the SG cohort as there was one mortality $(3.4 \%)$ compared to four in the noninvasive cohort (8.2\%). In order to assess long term outcomes, the authors examined patients who were able to follow up in 3 years (LT alone $n=36$ vs LT+SG $n$ =13). There was a significant maintenance in weight loss between BMI at listing and at 3 year follow up in the SG group $(49.0+/-$ 4.6 vs. $30.9+/-13.2$ ) respectively. In the noninvasive group, BMI at listing and last follow up showed no significant changes $(40.06$ 
+/- 2.99 vs. $38.53+$ +- 6.53) respectively. Complications related to SG included gastric staple line leak and bleeding which required re-operation. Overall obesity related complications in the SG and LT group showed lower prevalence at 3 years when compared to the noninvasive group. This was demonstrated as improved overall cardiovascular risk factors and less patients requiring medications for DM, HTN and dyslipidemia [28].

In summary, studies on simultaneous BS and LT show improvement in obesity related complications and results in more effective and durable weight loss. A further advantage of combined BS and LT surgery is the elimination of two separate procedures when compared to BS prior or after LT. On the other hand, BS and LT carry post-operative risk and careful selection of patients is important for the procedure's safety and feasibility. Moreover, these studies are limited in their sample size and duration of follow up. Further studies are also needed to assess the effects on immunosuppressive medications and graft survival.

\section{BS after LT}

Initiation of immunosuppressive therapy following LT coupled with decreased activity levels following this major surgery can lead to onset or worsening of the metabolic syndrome [29,30]. This worsening has negative effects on both quality of life and decreases transplant recipient survival and possibly graft function in morbidly obese [31]. For transplant patients with history of cirrhosis secondary to NASH, recurrence following LT is related to post-transplant BMI [32] and can be particularly severe [45]. Also, with increased lifespan for transplant patients, the sequalae of metabolic disease become a life-limiting factor for this population [31,33,34]. Bariatric surgery (BS) has been used to good effect for weight-loss with associated improvement in related comorbidities in the non-transplant population [35]. Post-transplant patients carry increased surgical risk due to immunosuppression with increased infection rates, decreased healing [36,37] and more complicated peritoneal access due to presence of adhesions from transplantation. We review six studies where BS was performed on patients with previous LT. Overall 43 patients were assessed for mortality, complications, percent excess weight loss (\%EWL), resolution of metabolic disease and immunosuppressive regimen.

No early mortality was reported with respect to BS (within 90 day of procedure), however 3 cases $(7 \%)$ of delayed mortality are reported. Al- Nowaylati., et al. [38] reports mortality in two patients at 6 months and 9 months from Fournier's Gangrene with history of penile prosthesis in situ and esophageal cancer, respectively. Osseis., et al. [39] reports a death at 19 months after laparoscopic sleeve gastrectomy (LSG) due to complications from a gastric staple line leak, ultimately leading to multi-organ failure.

Complications following BS included three wound infections [38,39], 1 anastomotic leak in Roux-en-Y gastric bypass (RYGB) requiring reoperation [40], 1 intraoperative bleed requiring splenectomy and portal vein thrombosis in the same patient [41], 3 with incisional hernias following open RYGB [38,42], 1 reversal of open RYGB due to malnutrition and a recalcitrant duodenal ulcer [38], 1 bile leak [42], dysphagia requiring conversion to RYGB [42], 3 with poor PO intake requiring pneumatic dilation and one with gastrostomy tube (required for oral immunosuppressive mediations) [43] and 1 late drain removal [43]. Altogether, fifteen patients of 43 (35\%) experienced complications related to BS. This aggregate complication rate remains well above rates reported in the nontransplant population [44]. Contributing factors are likely poor healing secondary to immunosuppressive regimens, increased risk of incisional hernia, presence of adhesions from previous transplant surgery, increased chance for infection due to immunosuppression and presence of foreign objects from previous transplant surgeries and repairs such as mesh for prior incisional hernia repair.

Effects on immunosuppression have been a concern unique to the post-transplant population with some apprehension regarding acute graft failure with decreased absorption of immunosuppressive medication. Among the studies reviewed, five reported the immunosuppressive regimens prior to and following BS. One reported no changes [43], one improved the amount of time of tacrolimus within therapeutic range from $39 \%$ to $47 \%$ without change in dosing [40], one required reduction in dosing [41], two studies reported increased dosing of immunosuppression [38,42]. No cases of acute graft rejection were reported.

Surgical approaches in patients undergoing BS are varied. Overall, 32 patients (74\%) underwent sleeve gastrectomy with a majority being via laparoscopic approach. Al-Nowaylati., et al. [38] performed open RYGB on all 7 of their patients, citing a concern for adhesions likely to be present in post-transplant patients. Yemini., 
et al. [40] used both LSG and LRYGB, though they do not account for how many of the LT patients underwent each procedure. They conclude that since stability of immunosuppressive regimens is present in both LSG and LRYGB, LSG may actually be less desirable than LRYGB in the long term, due to chronic leaks along the major gastric curvature in SG. On the other hand, many authors have cited the need to maintain endoscopic access to the biliary system as a reason to favor SG over RYGB. Further longitudinal studies would be needed to determine the rate of biliary access required in post RYGB patients and to further assess morbidity and mortality benefits in maintaining biliary access.

Regarding weight-loss and improvement in metabolic comorbidities, five of the studies reported a mean \%EWL which ranged from 46.5 to 76 at 12 months. Yemini., et al. reported a \%EWL of $69 \%$ for LSG and 75\% for LRYGB at 1 year from BS. Another group compared weight loss between transplant vs nontransplant patients undergoing LSG and showed 55\% EWL in transplant patients and 50\% EWL in non-transplant patients. Osseis., et al. [39] reported the highest \%EWL of 76 at 12 months post SG while Khoraki., et al. [41] reported \%EWL of 46.5, also all with patients undergoing SG. All 6 groups reported improvement in the metabolic comorbidities following BS with some patients able to achieve complete remission for diabetes, OSA, HTN, hyperlipidemia and NASH. Tsamalaidze., et al. [43] compared resolution of metabolic comorbidities between transplant and non-transplant patients undergoing BS. They found that resolution of diabetes, cardiac disease, hypertension, obstructive sleep apnea and hyperlipidemia occurred at similar rates between the two groups. Resolution of nonalcoholic steatohepatitis in their study could not be assessed as none of the LT patients had NASH at onset.

The long-term effect of BS on graft function is yet to be determine and longitudinal trials are needed to further inform whether the increased surgical risks in the LT population are acceptable in relation to benefit derived from BS for treatment of metabolically related comorbidities. While complication rates are high in the post LT population, improvement in patient selection may be able to mitigate this risk. It will also be important, in the future, to study not only durability of graft function and safety of bariatric procedures but also quality of life outcomes in post LT bariatric patients to help guide appropriateness of BS.

\section{Conclusion}

Bariatric surgery seems to be a safe option for patients with ESLD undergoing liver transplant regardless of the timing of the procedure in relation to the LT surgery. However, more studies are needed to evaluate the effect of nutritional status following RYGB on the outcome of LT surgery. Further studies are needed to assess the long-term effect of BS on graft function and immunosuppression medications.

\section{Bibliography}

1. Kelly T., et al. "Global burden of obesity in 2005 and projections to 2030". International Journal of Obesity 32.9 (2008): 1431-1437.

2. Younossi Z., et al. "Global burden of NAFLD and NASH: trends, predictions, risk factors and prevention". Nature Reviews Gastroenterology and Hepatology 15.1 (2017): 11-20.

3. Martin P., et al. "Evaluation for liver transplantation in adults: 2013 practice guideline by the American Association for the Study of Liver Diseases and the American Society of Transplantation". Hepatology 59.3 (2014): 1144-1165.

4. Barone M., et al. "Systematic review with meta-analysis: postoperative complications and mortality risk in liver transplant candidates with obesity". Alimentary Pharmacology and Therapeutics 46.3 (2017): 236-245.

5. Watt K. "Extrahepatic implications of metabolic syndrome". Liver Transplantation 19.2 (2013): S56-S61.

6. Lassailly G., et al. "Bariatric Surgery Reduces Features of Nonalcoholic Steatohepatitis in Morbidly Obese Patients". Gastroenterology 149.2 (2015): 379-388.

7. Buchwald $\mathrm{H}$ and Williams S. "Bariatric Surgery Worldwide 2003”. Obesity Surgery 14.9 (2004): 1157-1164.

8. Rogers C., et al. "Pharmacokinetics of mycophenolic acid, tacrolimus and sirolimus after gastric bypass surgery in endstage renal disease and transplant patients: a pilot study". Clinical Transplantation 22.3 (2007): 281-291.

9. Diwan T., et al. "Pharmacokinetic and pharmacogenetic analysis of immunosuppressive agents after laparoscopic sleeve gastrectomy". Clinical Transplantation 31.6 (2017): e12975. 
10. Diwan T., et al. "Liver Transplantation and Bariatric Surgery: Timing and Outcomes". Liver Transplantation 24.9 (2018): 1280-1287.

11. Dziodzio T., et al. "The Role of Bariatric Surgery in Abdominal Organ Transplantation-the Next Big Challenge?" Obesity Surgery 27.10 (2017): 2696-2706.

12. Sharpton S., et al. "Outcomes of Sleeve Gastrectomy in Obese Liver Transplant Candidates". Liver Transplantation 25.4 (2019): 538-544.

13. Lin M., et al. "Laparoscopic sleeve gastrectomy is safe and efficacious for pretransplant candidates”. Surgery for Obesity and Related Diseases 9.5 (2013): 653-658.

14. Shimizu H., et al. "Bariatric surgery in patients with liver cirrhosis”. Surgery for Obesity and Related Diseases 9.1 (2013): $1-6$.

15. Moulla Y., et al. "Feasibility and Safety of Bariatric Surgery in High-Risk Patients: A Single-Center Experience". Journal of Obesity (2018): 1-6.

16. Rebibo L., et al. "Laparoscopic sleeve gastrectomy in patients with NASH-related cirrhosis: A case-matched study". Surgery for Obesity and Related Diseases 10.3 (2014): 405-410.

17. Takata M., et al. "Laparoscopic bariatric surgery improves candidacy in morbidly obese patients awaiting transplantation". Surgery for Obesity and Related Diseases 4.2 (2008): 159-164.

18. Safwan M., et al. "Outcome of liver transplantation in patients with prior bariatric surgery". Liver Transplantation 23.11 (2017): 1415-1421.

19. Idriss R., et al. "Impact of Prior Bariatric Surgery on Perioperative Liver Transplant Outcomes”. Liver Transplantation 25.2 (2019): 217-227.

20. Chmura A. "The First Polish Liver Transplantation after Rouxen-Y Gastric Bypass Surgery for Morbid Obesity: A Case Report and Literature Review". Annals of Transplantation 20 (2015): 112-115.

21. Taneja S., et al. "Single-Lobe Living Donor Liver Transplant in a Morbidly Obese Cirrhotic Patient Preceded by Laparoscopic
Sleeve Gastrectomy". Case Reports in Transplantation (2013): $1-2$.

22. Campsen J., et al. "Adjustable Gastric Banding in a Morbidly Obese Patient During Liver Transplantation". Obesity Surgery 18.12 (2008): 1625-1627.

23. Kumar S., et al. "Simultaneous living donor liver transplant with sleeve gastrectomy for metabolic syndrome and NASHrelated ESLD-First report from India”. Indian Journal of Gastroenterology 36.3 (2017): 243-247.

24. Tariciotti L., et al. "Combined liver transplantation and sleeve gastrectomy for end-stage liver disease in a bariatric patient: First European case-report". International Journal of Surgery Case Reports 28 (2016): 38-41.

25. Guerrero Pérez F., et al. "Combined sleeve gastrectomy and orthotopic liver transplantation in patient with type 2 obesity and advanced liver disease". Endocrinología, Diabetes y $\mathrm{Nu}$ trición 64.5 (2017): 282-284.

26. Nesher E., et al. "Simultaneous Liver Transplantation and Sleeve Gastrectomy: Prohibitive Combination or a Necessity?" Obesity Surgery 27.5 (2017): 1387-1390.

27. Heimbach J., et al. "Combined Liver Transplantation and Gastric Sleeve Resection for Patients With Medically Complicated Obesity and End-Stage Liver Disease". American Journal of Transplantation 13.2 (2012): 363-368.

28. Zamora-Valdes D., et al. "Long-term outcomes of patients undergoing simultaneous liver transplantation and sleeve gastrectomy". Hepatology 68.2 (2018): 485-495.

29. Parikh N., et al. "Projected increase in obesity and non-alcoholic-steatohepatitis-related liver transplantation waitlist additions in the United States". Hepatology (2018).

30. Richards J., et al. "Weight gain and obesity after liver transplantation". Transplant International 18.4 (2005): 461-466.

31. Laish I., et al. "Metabolic syndrome in liver transplant recipients: Prevalence, risk factors, and association with cardiovascular events". Liver Transplantation 17.1 (2011): 15-22.

32. Nair S. "Obesity and its effect on survival in patients undergoing orthotopic liver transplantation in the United States". Hepatology 35.1 (2002): 105-109. 
33. Dureja P., et al. "NAFLD Recurrence in Liver Transplant Recipients". Transplantation 91.6 (2011): 684-689.

34. Pruthi J. "Analysis of causes of death in liver transplant recipients who survived more than 3 years". Liver Transplantation 7.9 (2001): 811-815.

35. Schauer P., et al. "Bariatric Surgery versus Intensive Medical Therapy in Obese Patients with Diabetes". New England Journal of Medicine 366.17 (2012): 1567-1576.

36. Andalib A., et al. "Early Postoperative Outcomes of Primary Bariatric Surgery in Patients on Chronic Steroid or Immunosuppressive Therapy". Obesity Surgery 26.7 (2015): 14791486.

37. Bootun R. "Effects of immunosuppressive therapy on wound healing". International Wound Journal 10.1 (2012): 98-104.

38. Al-Nowaylati A., et al. "Gastric bypass after liver transplantation”. Liver Transplantation 19.12 (2013): 1324-1329.

39. Osseis M., et al. "Sleeve Gastrectomy After Liver Transplantation: Feasibility and Outcomes". Obesity Surger 28.1 (2017): 242-248.

40. Yemini R., et al. "Bariatric surgery in solid organ transplant patients: Long-term follow-up results of outcome, safety, and effect on immunosuppression". American Journal of Transplantation 18.11 (2018): 2772-2780.

41. Khoraki J., et al. "Feasibility and outcomes of laparoscopic sleeve gastrectomy after solid organ transplantation". Surgery for Obesity and Related Diseases 12.1 (2016): 75-83.

42. Lin M., et al. "Safety and feasibility of sleeve gastrectomy in morbidly obese patients following liver transplantation". Surgical Endoscopy 27.1 (2012): 81-85.

43. Tsamalaidze L., et al. "Laparoscopic Sleeve Gastrectomy for Morbid Obesity in Patients After Orthotopic Liver Transplant: a Matched Case-Control Study". Obesity Surgery 28.2 (2017): 444-450.

44. Hutter M., et al. "First Report from the American College of Surgeons Bariatric Surgery Center Network". Annals of Surgery 254.3 (2011): 410-422.
45. Vallin M., et al. "Recurrent or de novo nonalcoholic fatty liver disease after liver transplantation: Natural history based on liver biopsy analysis". Liver Transplantation 20.9 (2014): 1064-1071.

\section{Assets from publication with us}

- Prompt Acknowledgement after receiving the article

- Thorough Double blinded peer review

- Rapid Publication

- Issue of Publication Certificate

- High visibility of your Published work

Website: www.actascientific.com/

Submit Article: www.actascientific.com/submission.php

Email us: editor@actascientific.com

Contact us: +919182824667 